\title{
Impacts of Air Interface Call Admission Control on UTRAN Transport Simulations
}

\author{
Yi Chen, Xi Li, Richard Schelb, Carmelita Görg and Andreas Timm-Giel
}

\author{
Yi Chen, Xi Li, Carmelita Görg and Andreas Timm-Giel \\ TZI-ikom, Communication Networks, University of Bremen, Germany, \\ e-mail: chenyi|xili|cg|atg@comnets.uni-bremen.de \\ Richard Schelb \\ Nokia Siemens Networks GmbH \& Co. KG, Germany, e-mail: richard.schelb@nsn.com
}

\begin{abstract}
This paper investigates the potential impacts caused by Call Admission Control (CAC) mechanism of the air interface on simulations carried out for the transport network domain of UMTS Radio Access Network. Independent from the TNL CAC mechanism of the transport network which is in charge of Iub link bandwidth, the Radio CAC function deals with the highly non-linear resources needed for an attempted connection depending on its specific propagation and interference situation. The consideration of the air interface acting as bottleneck results into a less challenging amount of traffic for the transport domain. In this work, a simulation model with Radio CAC mechanism is implemented to study this correlation, and evaluate the UTRAN transport network performance given a limited radio capacity. The analysis on the impact of the Radio CAC is derived from qualitative simulations.
\end{abstract}

Key words: UMTS, UTRAN, Iub, CAC, RRM, Dimensioning

\section{Introduction}

In Wideband Code Division Multiple Access (WCDMA) networks like Universal Mobile Telecommunication System (UMTS) network, Call Admission Control (CAC) plays an essential role for the network capacity allocation and prevents the network from being overloaded. Consequently, it guarantees Quality of Service (QoS) requirements of different types of services. As one of the important Radio Resource Management (RRM) functions, CAC is being used to protect the ongoing connections by limiting the capacity utilization below a certain level [1].

As specified in 3GPP [2], in addition to the CAC at the air interface (between UE and NodeB), there is also admission control required at the transport network of UMTS Terrestrial Radio Access Network (UTRAN), especially at the Iub interface (between NodeB and RNC) where the transport resource is scarce. Accordingly, the terms of Radio CAC and Transport Network Layer (TNL) CAC are used in the 
rest of this paper for the CAC algorithm locating at the air interface to protect the radio resource and at the Iub interface for the protection of ATM link bandwidth, respectively.

So far a lot of research has been ongoing to study the impact of CAC algorithms. [3] [4] present a work of estimation the required radio capacity of WCDMA in downlink, and [5] investigates the effects of the TNL CAC algorithm on Iub dimensioning. But the work from the current research is lack of investigating the effects caused by the coexistence of Radio CAC and TNL CAC. Therefore, a UMTS simulation model with both Radio CAC and TNL CAC is implemented in OPNET modeler and their impacts on the UTRAN transport network performance are studied in this paper.

The rest of the paper is structured as follows: Sect. 2 introduces the system model, including the principles of Radio CAC algorithm and examples of how to estimate the required transmission power in downlink at NodeB; The simulation results and analysis are presented in Sect. 3, where the impacts of Radio CAC are sensitively studied; finally, conclusions are given.

\section{System model}

\subsection{Model Overview}

CAC algorithm is composed of TNL CAC and Radio CAC, which manage the transport and radio resources respectively. The transport resource at the Iub interface is the allocated link bandwidth and the radio resource is represented in the total transmission power of base station. TNL CAC function is applied in UTRAN transport network to control the traffic load entering the Iub interface [5]. At the transport layer, each new Dedicated Channel (DCH) connection reserves certain bandwidth in order to protect the QoS. TNL CAC will check whether there is still enough bandwidth for this connection by comparing of the offered Iub link bandwidth with the sum of required bandwidth of this new connection and the already allocated bandwidth. If the requirement of a new connection exceeds the offered Iub link bandwidth, it will be rejected by TNL CAC, and no new connections are allowed until the link resource is released by other admitted connections.

Beside TNL CAC, another limiting factor of CAC is the radio interference, when considering a WCDMA radio interface. An interference-based admission control strategy, Radio CAC is introduced in the UTRAN model, which estimates the power increment caused by the requested radio access bearer and limits the total power consumption not over than the transmission power of base station.

Usually, the downlink capacity is expected to be more important than the uplink capacity because of a majority of asymmetric downloading type of traffic in UMTS. Therefore, within this work only downlink interference-based CAC is implemented. 
Assuming each NodeB in this UTRAN model has a maximum transmission power, which indicates its radio capacity. The power consumption caused by a connection request is from two aspects: one is the intercellular interference from adjacent cells, as variable scrambling codes are not as "orthogonal" as the own-cell signal; the other one is the power needed to overcome path loss. To avoid this overloading radio CAC needs to check that power increase caused by the admittance will not exceed the predefined limitation before admitting a connection request. Once the required power is over the predefined level, the connection request will be rejected by Radio CAC.

To estimate the power increase of a connection request, two steps are needed. One is to estimate power consumption as a function of interference; the other one is to find an appropriate propagation model to evaluate the path loss. These two steps are explained in the following part.

\subsection{Estimation of Required Transmission Power of NodeB}

Firstly, it starts from the assumption that due to the fast power control the UEs are able to obtain exactly the minimum average $E_{b} / N_{o}$ required for the service. It also assumes that UEs are uniformly distributed in each cell and thus cells are equally loaded, and the total transmit power of different NodeBs is equal. The generic link quality equation [3] for the downlink connection $i$ is expressed by:

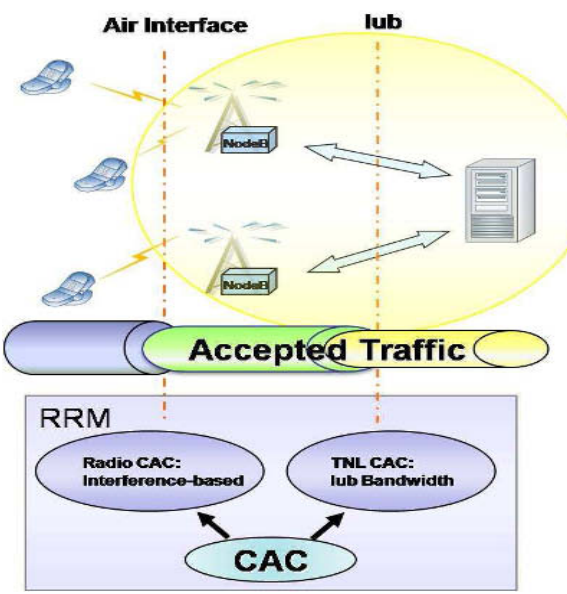

Fig. 1 shows the location of CAC algorithm. The first restriction of the system is from radio interface. Only the traffic accepted by the radio interface is the offered load of access network. After that, TNL CAC manages the Radio CAC allowed traffic and controls the traffic into access network. Therefore, the remaining traffic in the access network is the admitted part of TNL CAC. 


$$
\rho_{i}=\frac{W p_{i} / L_{m, i}}{R_{i}\left(\left(1-\alpha_{i}\right) P / L_{m, i}+P \sum_{n=1, n \neq m}^{N} 1 / L_{n, i}+P_{N}\right)}, i=1, \ldots, I .
$$

The description of the parameters in Equ. 1 is shown in the table below:

Table 1 Parameters used in generic link quality equation in downlink

\begin{tabular}{ll}
\hline Parameter & Description \\
\hline$p_{i}$ & $\begin{array}{l}\text { The required transmit power at NodeB } m \text { for the connection } i, i=1, \ldots, I \\
\text { Where } I \text { is the number of radio link }(\mathrm{RL}) \text { connections in the cell. }\end{array}$ \\
\hline$P_{N}$ & The thermal noise power, which is set to $-101.2 \mathrm{dBm}$ in this work. \\
\hline$R_{i}$ & The bit rate, determined by RAB rate of different service type. \\
\hline$W$ & The chip rate, in this work, WCDMA chip rate $W=3.84 M c p s$. \\
\hline$L_{m, i}$ & The required total transmission power of the NodeB, which indicates the radio \\
& capacity. \\
\hline$L_{n, i}$ & The path loss from the serving NodeB $m$ to UE $i$. \\
\hline$\rho_{i}$ & The path loss from another NodeB $n$ to UE $i$. \\
\hline$v_{i}{ }^{1}$ & $\begin{array}{l}\text { The } E_{b} / N_{o} \text { requirement for the UE } i, \text { including the SHO combining gain and } \\
\text { the average power raise caused by fast power control. }\end{array}$ \\
\hline$\alpha_{i}$ & The number of relevant neighboring NodeBs. \\
\hline & $\begin{array}{l}\text { The effective channel activity for the UE } i, \text { which is set to value } 0.5 \text { for speech } \\
\text { and value } 1 \text { for data service. }\end{array}$ \\
\hline
\end{tabular}

1 appears only in later equations

To solve the Equ. 1 a function $p_{i}$ of an individual required transmit power at NodeB $m$ for the connection $i$ is obtained [3]. The total transmission power $P$ is a sum of the individual connection powers in a cell.

$$
P=\frac{P_{N} \sum_{i=1}^{I} \frac{\rho_{i} R_{i} v_{i}}{W} L_{m, i}}{1-\sum_{i=1}^{I}\left[\frac{\rho_{i} R_{i} v_{i}}{W}\left(\left(1-\alpha_{i}\right)+f_{D L, i}\right)\right]}, f_{D L, i}=\frac{L_{m, i}}{L_{n, i}}
$$

In order to simplify the simulation model, the other-to-own-cell interference ratio in downlink $f_{D L, i}$ and the orthogonality $\alpha_{i}$ can be defined as the average. Thus the Equ. 2 becomes:

$$
P=\frac{P_{N} \sum_{i=1}^{I} \frac{\rho_{i} R_{i} v_{i}}{W} L_{m, i}}{1-\sum_{i=1}^{I}\left[\frac{\rho_{i} R_{i} v_{i}}{W}\left((1-\alpha)+f_{D L}\right)\right]}
$$

From the above Equ. 3 the path loss $L_{m, i}$ from the serving NodeB $m$ to UE $i$ can be estimated by using an extended COST-231HATA model with the frequency band $1500<=f(\mathrm{MHz})<=2000$ [7] using a set of predefined parameters: working 
frequency $f$, distance $d$ between UE and antenna, base station antenna height $h_{\text {Base }}$ and the height of the mobile antenna $h_{\text {Mobile }}$.

Note that in this Radio CAC implementation the connection does not occupy any radio resource when it is in Common Channel $(\mathrm{CCH})$. That means the transmission power needed to overcome the interference caused by $\mathrm{CCH}$ is negligible.

In a summary, the parameters used to estimate the required transmission power of NodeB in downlink direction are the distance between UE and NodeB antenna, the required Radio Access Bearer (RAB) rate and its associated $E_{b} / N_{o}$ value, and channel activity for different services. All these parameters vary according to the changes of mobile environment.

\section{Simulation results and analysis}

\subsection{Simulation Configuration}

The simulation model developed in [6] is extended with Radio CAC algorithm. The simulation scenario is composed of one NodeB and one RNC and in between is the Iub interface, on which the system performance is investigated. Web users are simulated using the web traffic model with Pareto distributed page size of an average value 50kbyte. User data transmission uses HTTP1.1 over TCP Reno. The reading time between pages is exponentially distributed with a mean of 5 seconds. Http application is generated on a $2 \mathrm{Mbps}$ E1 link where $1.6 \mathrm{Mbps}$ is actually used for user plane data transfer.

An additional information UE location is needed in radio CAC. The location information is the distance between UE and its serving NodeB with a cell range of $(1 \mathrm{~km}, 5 \mathrm{~km}$.

In this radio CAC algorithm each NodeB supports three cells, and the cell index is equally distributed among the connected users. If the new connection is admitted, the cell index, together with UE location information are recorded since radio CAC deals with cell specific power and interference.

\subsection{Performance Metrics}

As the changing of RAB rate affects the required transmission power estimation $\mathrm{CAC}$ algorithm is considered when:

- Connection establishment

- Channel Type Switching (CTS) between DCH and FACH/RACH

- Bit Rate Adaptation (BRA)

- Handover (hard/soft handover) 
For evaluating radio CAC function and its impacts on the UTRAN network, the statistics Radio CAC reject connection ratio and TNL CAC reject connection ratio are defined as the number of connections rejected by the $\mathrm{CAC}$ algorithm over all over connection requests. For each different RAB request, it has its own Radio CAC and TNL CAC reject ratio.

Other metrics also need to be considered to evaluate the whole system performance, like the end-to-end application delay at application layer, packet discard ratio which reflects the transport layer performance and ATM link throughput as well.

\subsection{System Performance with Varying Radio Capacity}

The system performance is observed with a varying radio capacity from $35 \mathrm{dBm}$ to $100 \mathrm{dBm}$ under traffic load $40 \%, 50 \%$ and $70 \%$.

Reject ratios of Radio CAC and TNL CAC are shown in Fig. 2 presents the system capacity restriction is migrating from radio capacity to a limited Iub link bandwidth when the maximum transmission power of NodeB increasing from $35 \mathrm{dBm}$ to $100 \mathrm{dBm}$. Higher reject ratio of CAC means less traffic is allowed to enter into the access network. Therefore, most traffic is rejected by Radio CAC at the point of $35 \mathrm{dBm}$ transmission power as radio capacity is quite low and limits the traffic. Since the traffic is mainly constrained by Radio CAC at the air interface, the Iub link capacity is relatively large thus no traffic is rejected by TNL CAC.

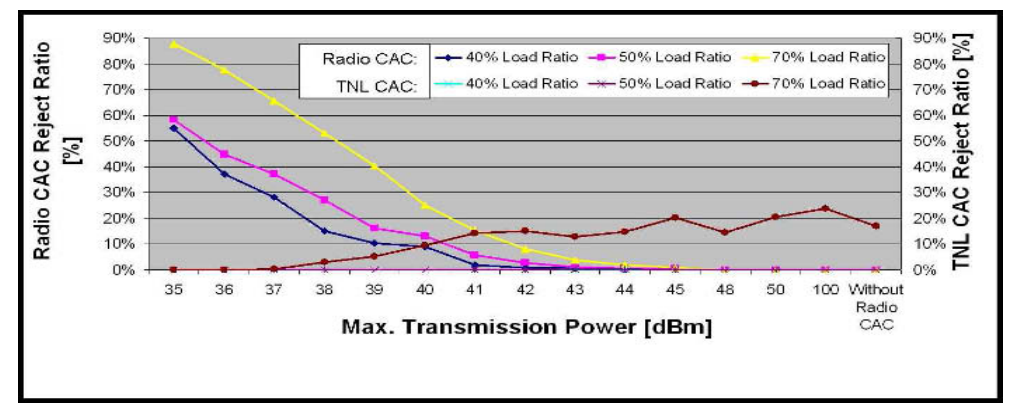

Fig. 2 Reject Ratios of Radio CAC and TNL CAC 
High Radio CAC reject ratio leads less traffic in the transport network, thus the transport layer performance is better due to less congestion, consequently, a higher application delay at application layer. Fig. 3(a) and Fig. 3(b) illustrate the system performance at application layer and transport layer respectively.

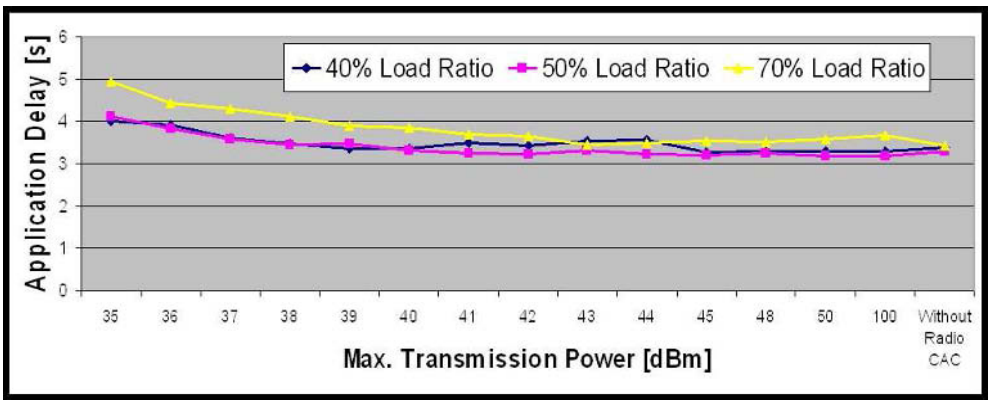

(a) Application delay at application layer

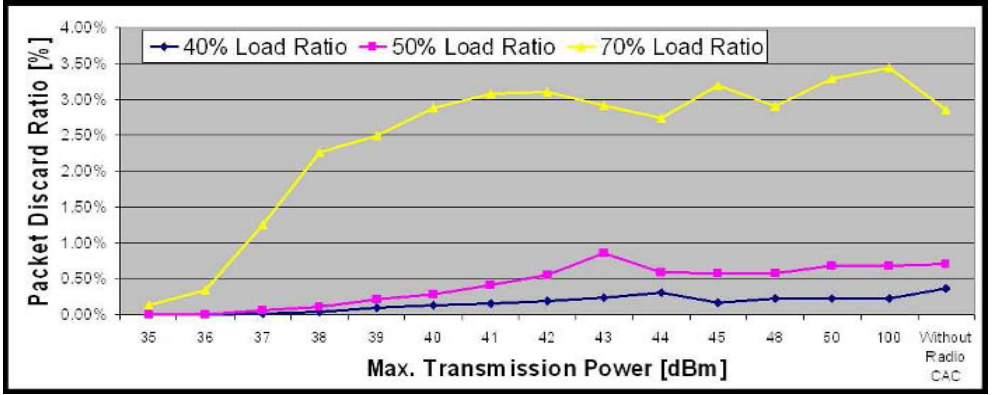

(b) Packet discard ratio at transport layer

Fig. 3 System performance at application layer and transport layer

In spite of the variation of traffic load, application delay in Fig. 3(a) has the same tendency that a long application delay is caused by low radio capacity and it decreases when transmit power is increased from $35 \mathrm{dBm}$ to $41 \mathrm{dBm}$. However, the application delay does not substantially decrease afterwards since the restriction moves from radio capacity to Iub link capacity. As the Iub link capacity is fixed, the application delay is in a stable state.

The long application delay means the requested application data are transmitted with a low speed in the lower layers. By comparing the RAB ratio in Tab. 2 with maximum transmission power $35 \mathrm{dBm}$ and $50 \mathrm{dBm}$ respectively, we can see that a low radio capacity lower $\mathrm{RAB}$ rate sets are used for the data transferring as more transmission power is required when switching to higher RAB rates. This causes the data transferring using lower RAB rates, e.g. ratio of RAB type $P S 8 / 8$ is $9.96 \%$ and $P S 64 / 256$ is $1.84 \%$ with $35 \mathrm{dBm}$ radio capacity, and they change to $0.00 \%$ and $10.16 \%$ respectively when radio capacity increases to $50 \mathrm{dBm}$. 
Table $2 \mathrm{RAB}$ rate ratios of maximum transmission power of $35 \mathrm{dBm}$ and $50 \mathrm{dBm}$

\begin{tabular}{lll}
\hline RAB Rate Ratio & Max. Transmission Power \\
$($ UL - rate $/ D L-$ rate $)[\mathrm{kbps}]$ & $35 \mathrm{dBm}$ & $50 \mathrm{dBm}$ \\
\hline PS 8/8 & $9.96 \%$ & $0.00 \%$ \\
PS 32/32 & $26.88 \%$ & $10.01 \%$ \\
PS 32/64 & $29.02 \%$ & $27.20 \%$ \\
PS 64/64 & $19.55 \%$ & $27.50 \%$ \\
PS 64/128 & $12.74 \%$ & $22.69 \%$ \\
PS 64/256 & $1.84 \%$ & $10.16 \%$ \\
PS 64/384 & $0.02 \%$ & $2.44 \%$ \\
\hline
\end{tabular}

As most traffic is blocked due to the lower RAB rates, there are no congestions in the transport layer between $35 \mathrm{dBm}$ and $41 \mathrm{dBm}$, where the transport layer performance is better. Fig. 3(b) shows the packet discard ratio at transport layer is lower than $3 \%$ when transmission power is not larger than $41 \mathrm{dBm}$.

When the radio capacity increases to a certain value, e.g. over $50 \mathrm{dBm}$, the limit caused by radio capacity is getting lower; on the other hand, TNL CAC starts to work as the Iub link capacity becomes the restriction. Therefore, within the region of both Radio CAC and TNL CAC working together, the performances of application layer, transport layer and ATM layer are changing little within a certain level. When the transmission power of NodeB is set to $100 \mathrm{dBm}$ which means there is no limit on the radio capacity, the overall performance of the system is similar to that of a system without Radio CAC.

\subsection{Correlation of Radio CAC and System Performance}

Based on the measurements in the Sec. 3.3, the association between Radio CAC reject ratio and system performance under different layers can be evaluated by calculating their correlation coefficient (c.c.) values. Taking an example of the correlation calculation between Radio CAC and application delay, the radio CAC reject ratio and application delay, two measurement sets are observed by varying radio capacity from $35 \mathrm{dBm}$ to $100 \mathrm{dBm}$. So the strength of linear association of these two measurement sets can be calculated using the standard measure correlation coefficient.

As illustrated in Fig. 4(a), from the sign of the c.c. values two RAB rate changing cases (new connection requesting and channel type switching) have a same linear relationship with the system performance. However the strength of association varies under different layers. The sign of the c.c. values represent the effects are positive or negative, i.e. when Radio CAC reject ratio increases it has a higher application delay, lower packet discard ratio at transport network and less ATM link throughput. In other words, higher Radio CAC reject ratio leads a worse performance in application layer but a better performance at transport layer. 


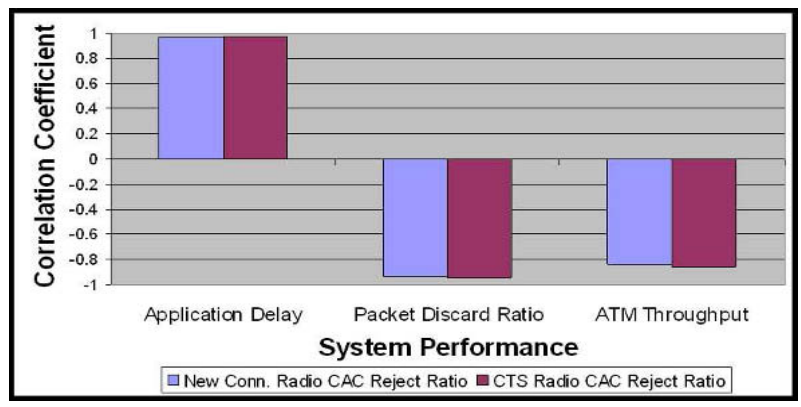

(a) Radio CAC and system performance

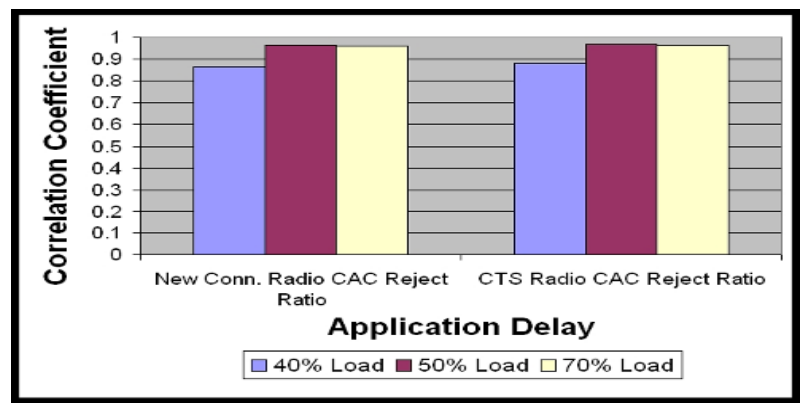

(b) Radio CAC and application delay

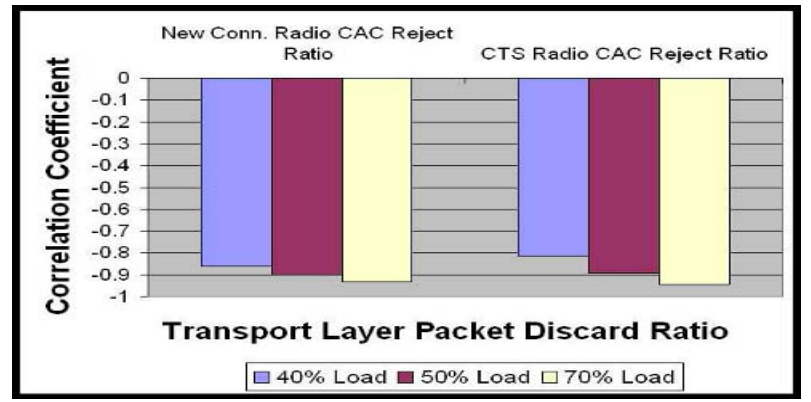

(c) Radio CAC and transport layer packet discard ratio

Fig. 4 Correlation coefficient values

If Radio CAC reject ratio increases, the application delay increases at the same time as they has positive c.c. value. That means the reduction of radio capacity will make the application performance worse as only lower RAB rate set is allowed. The contrast is the transport layer and ATM link performance which have negative c.c. values. The more radio capacity is offered, the more congestion is caused in lower layers. And transport layer is more sensitive to the Radio CAC reject ratio than ATM link layer, if we compare their c.c. values that transport layer has a c.c. value -0.9 which is smaller than the ATM link layer c.c. value -0.8 . 
Fig. 4(b) and Fig. 4(c) illustrate the correlation coefficient of Radio CAC with application delay and transport layer packet discard ratio under three different traffic load levels: $40 \%, 50 \%$ and $70 \%$. From these figures we can see although the relationship described above exists in different load levels, it varies as the load changes.

When the load level increases from $40 \%$ to $50 \%$, the variation of the effect from Radio CAC on application layer is significant. If the load level continues increasing to $70 \%$, the application performance does not have much change. The main reason is under high load, application performance is mainly restricted by Iub link capacity and at the same time the limit from radio capacity is not obvious.

Obviously, the influence of Radio CAC on transport layer is significant in Fig. 4(c), and increases when the load level increasing, since Radio CAC affects the selection of RAB rate directly in the transport layer.

\subsection{Compare with Dimensioning Results of Without Radio CAC}

In order to evaluate the impacts of Radio CAC on the UTRAN dimensioning results, the traffic load is simulated under different levels. The results of UTRAN dimensioning without Radio CAC are shown in blue curves which have recommended bandwidth where the QoS of Transport Network layer is satisfied, i.e. the transport layer packet discard ratio is less than $1 \%$. Based on the dimensioning results, Radio $\mathrm{CAC}$ is introduced with varying radio capacities, i.e. from $35 \mathrm{dBm}$ to $50 \mathrm{dBm}$. The system performance in terms of application, transport and ATM layer are observed in Fig. 5(a), Fig. 5(b) and Fig. 5(c).

Fig. 5(a) and Fig. 5(c) show that the impacts of Radio CAC are not significant in application and ATM layer at low traffic load. When the load increases, low radio capacity is the main restriction for the system that shown $35 \mathrm{dBm}$ has a highest application delay and lowest ATM link utilization. Although in Fig. 5(b) transport layer has lower packet discard ratios, it sacrifices the application and ATM layer performance. This means even the system has enough link bandwidth at Iub interface the relative small radio capacity is the main limit to the system performance. If the radio capacity increases to $50 \mathrm{dBm}$ the system performance will approach to the blue curve where the restriction moves to Iub link bandwidth. As it is seen from Fig. 5(c) the link utilization is identical when the maximum transmission power is set to $50 \mathrm{dBm}$. However, the application and transport layer performances are not identical, especially the packet discard ratio which is lower than the blue curve. This difference shows even the restriction of the system is from the Iub link bandwidth, the limit of radio capacity still can affect the selection of high RAB rates. This impact is shown in Fig. 5(a), application delay still increases while Iub bandwidth increasing to $5100 \mathrm{kbps}$, as data traffic is transmitted using lower RAB rates in transport layer. 


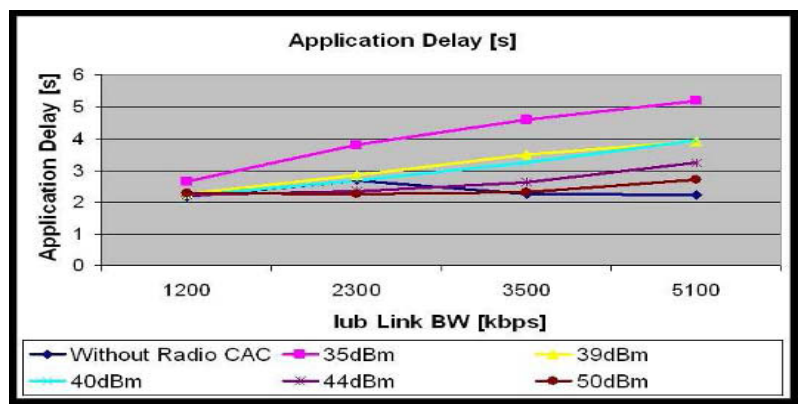

(a) Application delay

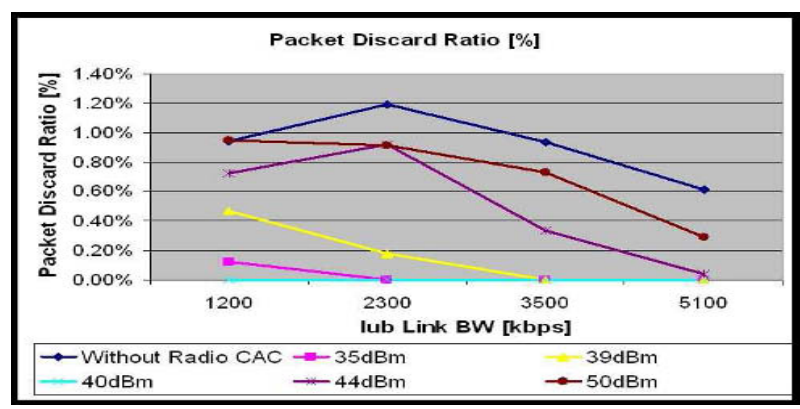

(b) Packet discard ratio

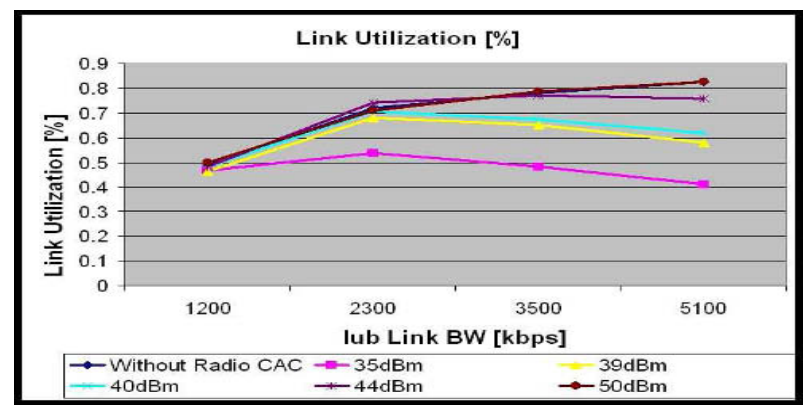

(c) Link utilization

Fig. 5 Comparison of dimensioning results

Base upon the analysis of system performance, Radio CAC controls the traffic offering onto the access network further limits the traffic bursty therefore has a better transport layer performance, however the application performance gets worse as data are transmitted using lower RAB rates. 


\section{Conclusion}

As another restriction to the system capacity, Radio CAC brings an additional bottleneck to the UTRAN network at the air interface with a limited radio capacity and is independent of TNL CAC at Iub interface. The introduction of Radio CAC to the system model controls the traffic and affects the selection of RAB rates when the BRA function is switched on. Since Radio CAC limits to select the higher RAB rates, data are transferred with lower speed, thus it has a higher application delay and less congestion in transport network domain. Additionally, the impacts are not identical in different layers. The experience shows it has more influences on transport layer than ATM layer as Radio CAC can affect the selection of RAB rates directly and the effects will increase along with the increase of traffic load. As it limits the data transferring speed by using lower RAB rates, transport layer performance are getting better. Therefore, by comparing the dimensioning results from the system without Radio CAC, the system with Radio CAC can achieve a better QoS in transport layer but with the sacrifice of a worse application performance.

Acknowledgements This work is carried out in the master thesis which is supported by the Nokia Siemens Networks Project Mature (Modeling and Analysis of the Transport Network Layer in the UTRAN Access Network REsearch). The partner of this work is Nokia Siemens Networks in Berlin, Germany.

\section{References}

1. Harri Holma, Antti Toskala: WCDMA for UMTS: Radio Access for Third Generation Mobile Communications. Wiley Technology Publishing, 1 edition (June 7, 2000)

2. 3GPP TS 25.401 v3.10.0, 3rd Generation Partnership Project; Technical Specification Group Radio Access Network; UTRAN Overall Description (Release 1999). Wiley Technology Publishing, 1 edition (June 7, 2000)

3. Kari Sipil, Zhi-Chun Honkasalo, Jaana Laiho-Steffens, Achim Wacker: Estimation of Capacity and Required Transmission Power of WCDMA Downlink Based on a Downlink Pole Equation. VTC 2000, P1002-1005, 2000

4. Chie Dou, Yu-Hua Chang: Class-based downlink capacity estimation of a WCDMA network in a multiservice context. Computer Communications 28 (2005) 1443-1455

5. Xi Li, Richard Schelb, Andreas Timm-Giel and Carmelita Görg: Dimensioning of UTRAN Iub Links for Elastic Internet Traffic with Multiple Radio Bearers. 13th GI/ITG Conference Measuring, Modelling and Evaluation of Computer and Communication Systems, Nrnberg, March 2006.

6. Thushara Weerawardane, Xi Li, Andreas Timm-Giel and Carmelita Görg: Modeling and Simulation of UMTS HSDPA in OPNET. OPNETWORK 2006, September, 2006, Washington DC, USA

7. Robert Akl, Manju Hegde, Alex Chandra: CCAP: CDMA Capacity Allocation and Planning. Washington University, Missouri, USA, April 1998 